\title{
Developing Assistant Tools for Geometric Camera Calibration: Assessing the Quality of Input Images
}

\author{
Jean-Nicolas Ouellet Patrick Hébert \\ Computer Vision and Systems Laboratory \\ \{jouellet,hebert\}@gel.ulaval.ca, Laval University, Québec, Canada
}

\begin{abstract}
This paper proposes two indicators for predicting the quality of camera model parameters from a set of input images. The first indicator is based on the acutance. It can quickly indicate static or motion blur during image capture and correlates well with the $3 D$ reconstruction error when using stereo cameras. The second indicator provides the overall distribution of control points in a set of input images. Although the importance of covering the entire field of view is verified, the spatial distribution of control points need not be uniform. The analysis is supported by experiments with mono and stereo cameras calibrated using both a low cost planar and a high quality $3 D$ target.
\end{abstract}

\section{Introduction}

Geometric camera calibration is commonly perceived as a laborious process including the steps of collecting a set of images from a calibration target, matching the image features (control points) to the target model and optimizing the camera model parameters. Initially developed and applied by photogrammetry specialists, camera calibration is now applied by various non experts in contexts that differ from optimal conditions met in usual photogrammetry applications. Images can be captured slightly out of focus or low cost cameras and lenses can be used; the camera can be calibrated hand-held or on a tripod possibly in the presence of ground vibrations. How good is the expected result? Since the quality of the camera model parameters rely on the quality of the input images, one needs reference indicators that can be quickly and easily interpreted. Based on the analysis of the input images, we aim at providing these indicators to help one decide whether new images should be captured before completing the whole procedure.

Commercial photogrammetric software or implementations of well-known calibration methods with available software are commonly used. The latter include Tsai's method developed in the 1980's [1], the method proposed by Zhang using a simple planar calibration target that is moved relatively to the camera [2], or the method proposed more recently by Heikkila [3]. In the best case, variance estimates for the camera model parameters are provided along with the projection errors. Nevertheless, these indices are not always simple to interpret since the model parameters are dependent and their distributions unknown.

In this paper, we show that these values may not be sufficient to confirm the quality of calibration. We concentrate our efforts on the analysis of input images. This analysis is based on the image sharpness and the coverage of control points in a set of images.

To reduce bias due to non uniform illumination, higher accuracy can be obtained by detecting feature boundaries with subpixel precision, and then fitting a geometric model to the observations [4]. Since sharper images lead to better control point positions, a simple analysis based on acutance can quickly reveal focus quality or motion blur. This is developed in sections 2 and 3. In section 4 the distribution of control points in the set of images is examined. When multiple images from different viewpoints are input to provide a better coverage, this distribution is generally irregular. Calibration experiments are carried out to study the influence of various distributions. The interpretation is supported by experiments with mono and stereo cameras calibrated using both a low cost planar and a high quality 3D target.

\section{Acutance}

Acutance is an objective measure of image sharpness. In the last 50 years, different formulae have been proposed to correlate well with subjective image sharpness [5]. In this section and the following, it is shown that acutance is a useful indicator for predicting camera calibration quality.

The acutance is measured from the intensity distribution across an image edge. Although an ideal edge model is a step edge, a real edge in an image has a gradually sloping profile. Image Edge Profile (IEP) acutance can be measured along the edge profile normal to an object boundary:

$$
I E P=\frac{1}{m} \sum_{i=1} \frac{f(i)-b(i)}{2 i},
$$




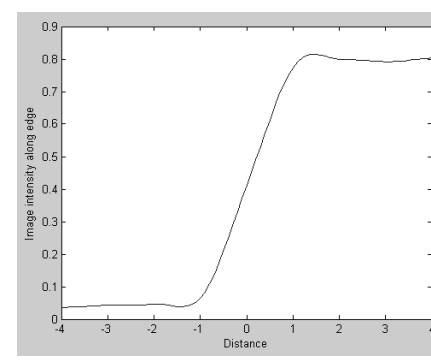

(a)

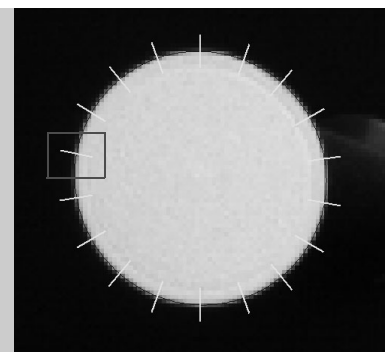

(b)
Figure 1. a) One edge profile sampled along the contour normal of the control point image in b).

where $f(i)$ and $b(i)$ are the foreground and background pixels on both sides of the edge position. Figure 1 a depicts such a profile along the image of a white circle on a black background. This control point image is a section of a calibration target image. The interval corresponding to a value of $m=4$ pixels along the profile, is drawn in b. In this case, the edge position is detected with subpixel accuracy (see [4] for details) and its orientation estimated. The image is then sampled along the edge normal using interpolation, bicubic in this case. To draw Figure 1 a, the profile was sampled at every 0,1 pixel.

From the IEP, one can further compute the average acutance, $A_{a v g}$, for a control point image and eventually, $\bar{A}_{a v g}$, for the whole set of control points in an image:

$$
A_{\text {avg }}=\frac{1}{d_{\text {max }}} \sqrt{\frac{1}{N} \sum \operatorname{IEP}(j)^{2}},
$$

where $d_{\text {max }}$ is the maximum value for IEP given an ideal step edge, and $\mathrm{j}$ indexes the $N$ edge pixels along the object contour. The height of the ideal step edge is estimated at a given distance from the edge position (at 5 pixels from the edge position in the illustrated example).

Since calibration targets are very contrasting - white targets on a black background or the converse - the IEP and $A_{\text {avg }}$ are particularly useful measures. Figures $2 \mathrm{a}$ and $\mathrm{b}$ compare the IEP acutance for two control point images where only the first is in focus. To draw the curves on the left, the IEP was estimated at every edge pixel along the ellipse contour resulting from the perspective projection of a circular target point. The parameterization is angular and varies between -180 and 180 degrees. The average acutance drops by $25 \%$ between both cases when $m=4$.

Besides a useful indicator of image sharpness, we show that it can also be an indicator of motion blurriness due to inappropriate acquisition conditions such as hand motion or ground vibration. Figures $2 \mathrm{c}$ and d show the interesting effect of very small and stronger motion blur on IEP. It can be observed that the motion along a direction leads to a characteristic signature in the image where the acutance

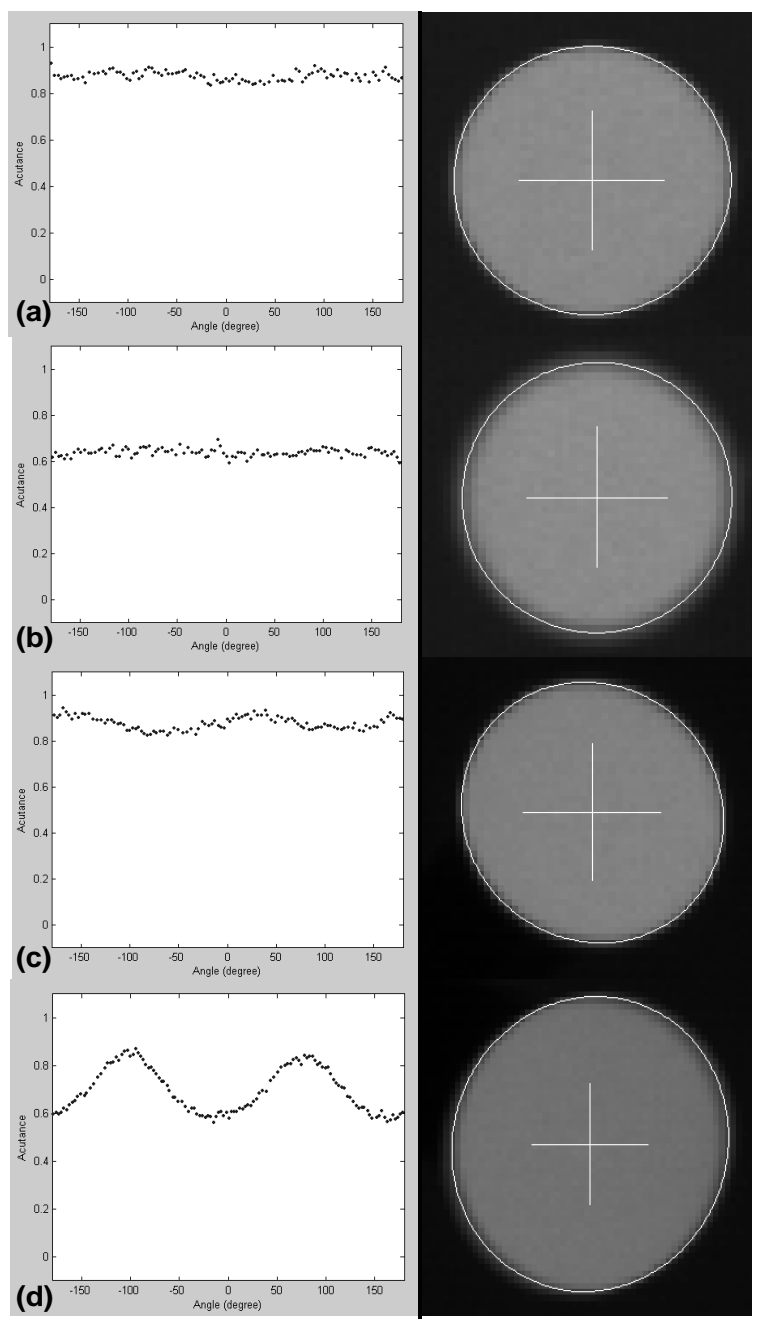

Figure 2. Acutance curve along a control point contour. a) Well focused image. b) Blurry image. c) Slight motion blur. d) Strong motion blur.

minima are reached along the motion direction. In Figure 2 c, the amplitude is 0,1 whereas it is nearly 0,25 in $\mathrm{d}$.

From these observations, we now devise an experiment that relates the acutance of the input images to the quality of the final camera model parameters. One can then obtain a feedback as soon as the input stage is completed.

\section{Experiment}

The experiment consists in calibrating two cameras while varying the focus. To simplify view collection a 3D target, measured using a CMM with micrometric accuracy ( $5 \mu \mathrm{m}$ for a $500 \mathrm{~mm}$ calibration target), is captured in a single frame using a stereo rig. We use a photogrammetric calibration model [3] where the intrinsic parameters include the coordinates of the principal point, the focal length, the pixel aspect ratio (scale factor), 2 radial and 2 
(a)

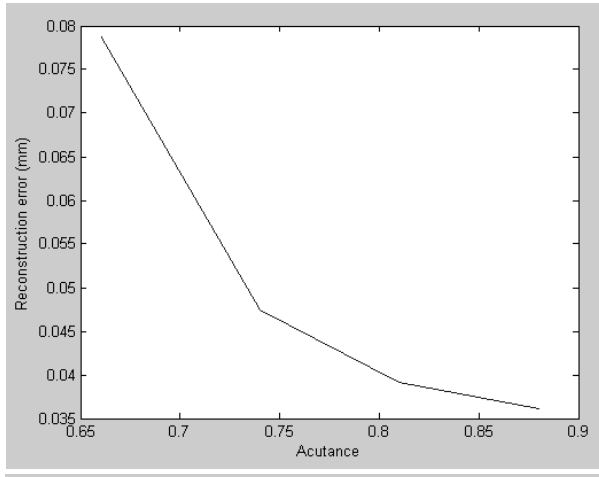

(b)

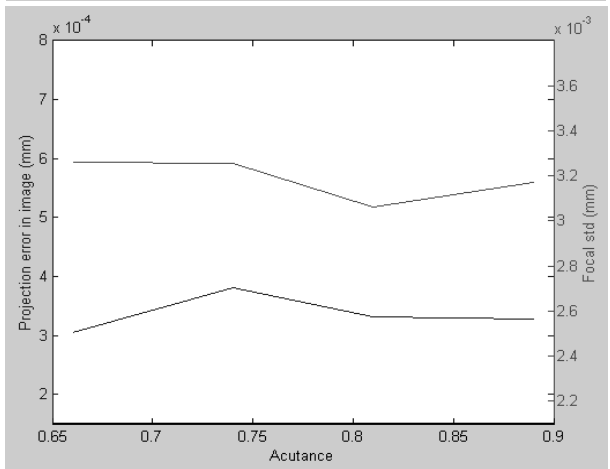

Figure 3. a) Average 3D reconstruction error vs. acutance. b) Average projection error (bottom curve, left scale) and focal length standard deviation (top curve, right scale) vs. acutance.

tangential distortion parameters, and the extrinsics describe the transformation between the two cameras.

To assess the quality of calibration, a second stereo frame is collected at a very close but different position in order to obtain an independent set of data. The 3D calibration target structure is recovered and aligned with its actual model. The alignment is optimal for a rigid transformation that minimizes the distance between the reconstructed target and the actual model. This 3D reconstruction error is the reference. Along with this error, the standard deviation for each of the estimated calibration parameters and the average projection error are calculated. This procedure is repeated 4 times at nearly the same relative position with respect to the calibration target but with varying focus adjustment for the camera lenses.

The results of this experiment are summarized in Figure 3 where the average (RMS) 3D reconstruction error is plotted against the acutance, $\bar{A}_{\text {avg }}$, in a. In Figure $3 \mathrm{~b}$, we have plotted the average projection error and the estimated standard deviation for the focal length of the left camera. The curves for the right camera lead to similar results. This experiment shows that the variation of acutance is highly correlated with the reconstruction error, i.e. the ground truth. This is in conformity with stereo triangulation where the depth precision is proportionnal to the image feature precision which is influenced by the focus. It is even better correlated than the average projection error or the parameter estimated standard deviation.

What is specific with the use of acutance? Actually, one might argue that collecting a large set of images for each camera position allows one to estimate the standard deviation of the control point positions and derive similar interpretation. This is true but acutance can be derived from a single image and can also indicate motion blurriness.

The average acutance for each of the input images indicate which images should be recaptured due to blurriness or motion blur. Conversely, one can adjust the focus of a camera using the objective measure provided by the acutance. Moreover, standard deviation of acutance among the control points in a same image is related to the depth of field. One thus gets a strong indicator before calibration parameters are computed.

\section{Image coverage}

Also obtained from the input images, the image coverage by the control points is a second indicator of the expected calibration quality. It is obviously preferable to capture control points everywhere in the image in order to better estimate camera model parameters. When calibration is performed from multiple viewpoints, we can easily superimpose the whole set of control points to observe their distribution. In practice, it is sometimes difficult to collect a uniform distribution. However, for typical cameras and lenses used in vision applications, the projection error due to distortion is stronger near the boundaries of the image. In this section, we examine this effect of image coverage on the estimation of the parameters, including the radial and tangential distortion parameters.

Since the correction provided by these parameters varies with the relative position to the principal point, it is proposed to examine their effect by partitioning the image into 5 areas including the center and the four corner areas (see Figures $4 \mathrm{c}$ and d). To do so, a single camera is calibrated using a set of 5 to 14 images captured from different positions and orientations. The experiment is repeated for two types of CCD cameras ( 0,5 inch analog and digital) mounted with short focal $(6 \mathrm{~mm})$ lenses. The experiment was also repeated for planar and 3D calibration targets. These calibration targets are illustrated in Figures $4 \mathrm{a}$ and $\mathrm{b}$ respectively. In both cases, the camera is calibrated and the projection error is analysed. The lower sections of the figure depict the distribution of all control points used in the multi-image set. The camera model parameters are estimated using different combinations of regions in the images. Table 1 illustrates the different cases for the planar target. The results for the 3D target are very similar, although not reproduced here. The first column lists the areas where the control points were used to estimate the parameters. The 5 following columns list the RMS values 
Table 1: Local variation of projection error in pixels due to partial coverage (planar target)

\begin{tabular}{|c|c|c|c|c|c|}
\hline $\begin{array}{c}\text { calibrate } \\
\text { using }\end{array}$ & 1 & 2 & 3 & 4 & 5 \\
\hline \hline all & 0,02 & 0,02 & 0,02 & 0,02 & 0,02 \\
\hline 1 & 0,01 & 0,05 & 0,05 & 0,05 & 0,05 \\
\hline $1,3,4$ & 0,02 & 0,05 & 0,02 & 0,02 & 0,04 \\
\hline $1,2,5$ & 0,02 & 0,02 & 0,04 & 0,04 & 0,02 \\
\hline $2,3,4,5$ & 0,03 & 0,02 & 0,02 & 0,01 & 0,02 \\
\hline
\end{tabular}

of the projection errors for each of the areas. The first row is the reference since all control points are used. In the table, the shaded cells highlight the variation of the projection error for the areas not considered for parameter estimation. It is clear that it is not only important to capture points in the corner regions but in all regions. Actually, using control points in only one image side significantly affects the error on the opposite side. It is also interesting to verify the influence of the relative number of points in the corner areas compared with the center area. To do so, we have progressively reduced the number of points in the corner areas by randomly selecting subsets in each area. In each case, the calibration experiment is repeated 30 times and both the RMS and the maximum projection error computed for all points, including those not used for calibration. Figure 5 illustrates the behavior for the planar target; a similar curve is observed for the 3D target. The curve shows that for a ratio, $r=\#$ corner/\#center, of at least $1 / 10$ th of the points in the corner areas, the projection error only converges slowly toward the control point position error

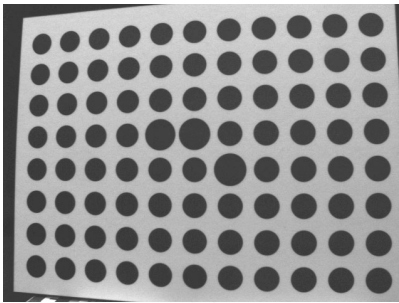

(a)

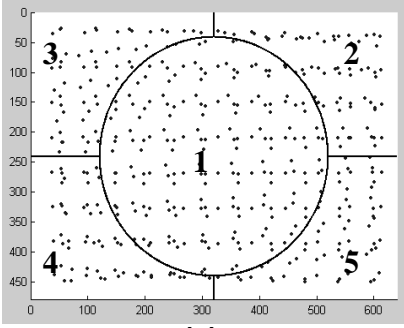

(c)

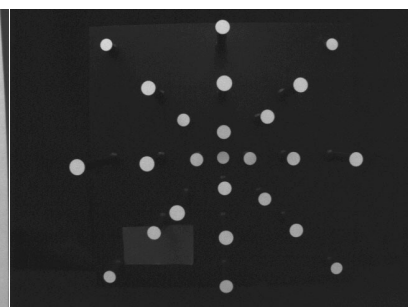

(b)

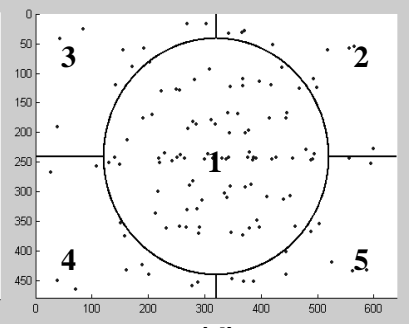

(d)
Figure 4. Coverage from 5 input images. a) Planar calibration target. b) 3D target. c) Coverage in the 5 areas: planar target. d) 3D target.
This is explained by the importance of distortion in the corner areas for these short focal lenses. This also means that sampling can be reduced in the corner areas without significantly affecting the results. For a given type of lens, a simple image such as in Figure $4 \mathrm{c}$ or $\mathrm{d}$ accompanied with the ratio $r$, thus provides a second useful indicator.

\section{Conclusion}

The two indicators based on the acutance and the image coverage depend only on the input images. They eliminate the needs to carefully examine each of the images and thus facilitate the calibration process. We have preferred not setting absolute thresholds but let the human operator interpret the objective measures (indicators). One can go further in the development of assistant tools for complex processes such as camera calibration. One way consists in examining the final parameters and their range of validity in space.

\section{References}

[1] R. Y. Tsai, "A Versatile Camera Calibration Technique for High-Accuracy 3D Machine Vision Metrology Using Offthe-Shelf TV Cameras and Lenses,' IEEE J. Robotics and Automation, Vol. 3, No. 4, Aug.1987, pp. 323-344.

[2] Z. Zhang, "A Flexible New technique for Camera Calibration", Tech. report MSR-TR-98-71, Microsoft research, Redmond, WA (USA), Dec. 2, 1998.

[3] J. Heikkilä, Geometric Camera Calibration Using Circular Control Points, IEEE Trans. on Pattern Analysis and Machine Intell., Vol. 22, No. 10. Oct. 2000, pp. 1066-1077.

[4] J. Heikkilä, "Moment and Curvature Preserving Technique for Accurate Ellipse Boundary Detection", Proc. 14th. Int'1 Conf. on Pattern Recognition, Aug. 1998, pp. 734-737.

[5] R. M. Rangayyan, N. M. El-Faramawy, J. E. Leo Desautels, and O. A. Alim, "Measures of Acutance and Shape for Classification of Breast Tumors", IEEE Trans. on medical Imaging, Vol. 16, No. 6, Dec. 1997, pp. 799-810.

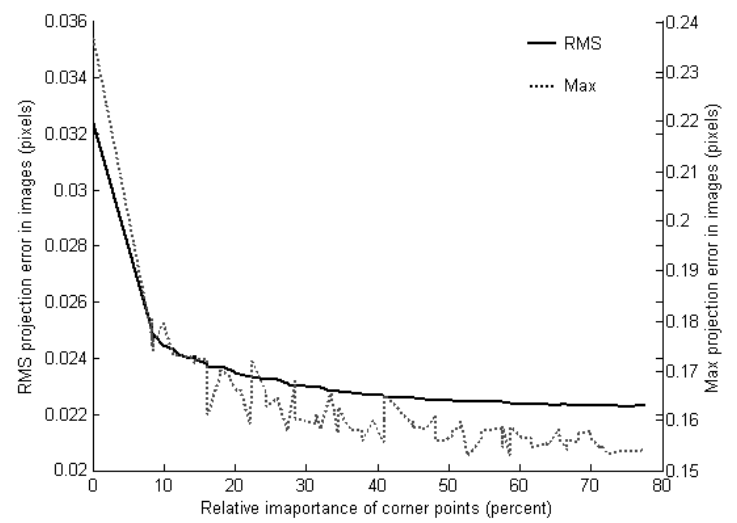

Figure 5. Influence of the relative number of control points in the corner areas (w.r.t. the center) on the RMS and maximum projection errors. 